MATHEMATICS OF COMPUTATION

Volume 67, Number 224, October 1998, Pages 1473-1491

S 0025-5718(98)00997-1

\title{
FAST SOLVERS OF INTEGRAL AND PSEUDODIFFERENTIAL EQUATIONS ON CLOSED CURVES
}

\author{
J. SARANEN AND G. VAINIKKO
}

\begin{abstract}
On the basis of a fully discrete trigonometric Galerkin method and two grid iterations we propose solvers for integral and pseudodifferential equations on closed curves which solve the problem with an optimal convergence order $\left\|u_{N}-u\right\|_{\lambda} \leq c_{\lambda, \mu} N^{\lambda-\mu}\|u\|_{\mu}, \lambda \leq \mu$ (Sobolev norms of periodic functions) in $\mathcal{O}(N \log N)$ arithmetical operations.
\end{abstract}

\section{Introduction: The PRoblem And the Purposes}

In this paper we deal with the problem

$$
\mathcal{A} u=f,
$$

where $f$ is a given 1-periodic function, $u$ is a 1-periodic function which we look for, and $\mathcal{A}=\sum_{p=0}^{q} A_{p}$ is a linear operator with $A_{p}$ of the form

$$
\begin{gathered}
\left(A_{0} u\right)(t)=\int_{0}^{1}\left[\kappa_{0}^{+}(t-s) a_{0}^{+}(t, s)+\kappa_{0}^{-}(t-s) a_{0}^{-}(t, s)\right] u(s) d s, \\
\left(A_{p} u\right)(t)=\int_{0}^{1} \kappa_{p}(t-s) a_{p}(t, s) u(s) d s, \quad p=1, \ldots, q .
\end{gathered}
$$

Here $a_{0}^{ \pm}(t, s)$ and $a_{p}(t, s)$ are $C^{\infty}$-smooth 1-biperiodic functions; $\kappa_{0}^{ \pm}(t)$ and $\kappa_{p}(t)$ are 1-periodic functions or distributions. We assume that the Fourier coefficients

$$
\begin{aligned}
& \hat{\kappa}_{0}^{ \pm}(m)=\int_{0}^{1} \kappa_{0}^{ \pm}(t) e^{-i m 2 \pi t} d t=\left\langle\kappa_{0}^{ \pm}(t), e^{-i m 2 \pi t}\right\rangle, \quad m \in \mathbb{Z}, \\
& \hat{\kappa}_{p}(m)=\int_{0}^{1} \kappa_{p}(t) e^{-i m 2 \pi t} d t=\left\langle\kappa_{p}(t), e^{-i m 2 \pi t}\right\rangle, \quad m \in \mathbb{Z}, p=1, \ldots, q,
\end{aligned}
$$

are known and satisfy the conditions

$$
\begin{gathered}
c_{1}|m|^{-\alpha} \leq\left|\hat{\kappa}_{0}^{+}(m)\right| \leq c_{2}|m|^{-\alpha}, \quad\left|\hat{\kappa}_{0}^{+}(m)-\hat{\kappa}_{0}^{+}(m-1)\right| \leq c|m|^{-\alpha-\beta}, \\
\hat{\kappa}_{0}^{-}(m)=\hat{\kappa}_{0}^{+}(m) \operatorname{sign}(m), \quad m \in \mathbb{Z}, m \neq 0 ; \\
\left|\hat{\kappa}_{p}(m)\right| \leq c|m|^{-\alpha-\beta}, \quad m \in \mathbb{Z}, m \neq 0, \quad p=1, \ldots, q,
\end{gathered}
$$

Received by the editor January 11, 1995 and, in revised form, July 22, 1996.

1991 Mathematics Subject Classification. Primary 65R20; Secondary 65N35, 45E10.

Key words and phrases. Boundary integral equation, trigonometric Galerkin method, fast algorithms. 
with some $\alpha \in \mathbb{R}, \beta>0$ and positive constants $c_{1}, c_{2}, c$. We also assume that

$$
\begin{gathered}
b_{1}(t):=a_{0}^{+}(t, t)+a_{0}^{-}(t, t) \neq 0, \quad b_{2}(t):=a_{0}^{+}(t, t)-a_{0}^{-}(t, t) \neq 0 \quad \forall t \in \mathbb{R}, \\
W\left(b_{1}\right)=W\left(b_{2}\right),
\end{gathered}
$$

where $W\left(b_{j}\right)=\left.\frac{1}{2 \pi} \arg b_{j}(t)\right|_{t=0} ^{t=1}$ is the winding number [15] of the function $b_{j}, j=$ 1,2 .

A variety of problems of type (1) - (6) arises from boundary integral equations on a smooth Jordan curve $\Gamma$ which is parametrized by a smooth 1-periodic function $t \rightarrow x(t): \mathbb{R} \rightarrow \Gamma$. For convenience of the reader we recall two specific examples in the following. For other examples we refer to [5], [8]-[10], [16].

Example 1.1. ([3], [20]) Consider the biharmonic single layer equation in the parameter form

$$
(\mathcal{A} u)(t):=\int_{0}^{1}|x(t)-x(s)|^{2} \log \left|x(t)-x(s) \| x^{\prime}(s)\right| u(s) d s=f(t) .
$$

We decompose the operator $\mathcal{A}$ as $\mathcal{A}=A_{0}+A_{1}$, where

$$
\begin{gathered}
\left(A_{0} u\right)(t)=\int_{0}^{1} \kappa(t-s) a(t, s) u(s) d s, \\
\kappa(t)=4 \sin ^{2} \pi t \log (2|\sin \pi t|), \\
a(t, s)=\left\{\begin{array}{lll}
\frac{|x(t)-x(s)|^{2}\left|x^{\prime}(s)\right|}{4 \sin ^{2} \pi(t-s)}, & t \neq s & (\bmod 1), \\
\frac{\left|x^{\prime}(t)\right|^{3}}{4 \pi^{2}}, & t=s & (\bmod 1),
\end{array}\right.
\end{gathered}
$$

and the operator $A_{1}$ has a smooth kernel, say $a_{1}(t, s)$. Function $\kappa$ has the Fourier coefficients

$$
\hat{\kappa}(m)= \begin{cases}\frac{1}{|m|\left(m^{2}-1\right)}, & |m| \geq 2, \\ -3 / 4, & |m|=1, \\ 1, & m=0 .\end{cases}
$$

In this example we have $\alpha=3, \kappa_{0}^{+}(t)=\kappa(t), a_{0}^{+}(t, s)=a(t, s), a_{0}^{-}(t, s) \equiv 0$. Moreover we can take $\beta=1$ since

$$
|\hat{\kappa}(m)-\hat{\kappa}(m-1)| \leq c|m|^{-4}, \quad m \neq 0,
$$

and $A_{1}$ has the form given in (2) with $\kappa_{1}(t) \equiv 1$ (implying that (5) is valid for all $\beta \in \mathbb{R})$. Since we have $a_{0}^{+}(t, t)=\left|x^{\prime}(t)\right|^{3} /\left(4 \pi^{2}\right), \quad a_{0}^{-}(t, t) \equiv 0$, the condition (6) is valid. Assuming (7), the operator $\mathcal{A}: H^{\lambda} \rightarrow H^{\lambda+3}, \lambda \in \mathbb{R}$, is an isomorphism.

Example 1.2. A large class of singular integral equations, see, e.g., [15], can be given in the parametric form as

$$
(\mathcal{A} u)(t):=a(t) u(t)+\int_{0}^{1} \kappa(t-s) b(t, s) u(s) d s=f(t),
$$

where $\kappa(t)=1+i \cot \pi t$ and the integral is understood in the sence of Cauchy. Writing

$$
a(t) u(t)=\int_{0}^{1} \delta(t-s) a(s) u(s) d s,
$$

where $\delta(t)$ is the 1-periodic Dirac distribution, we have $\mathcal{A}=A_{0}$ such that $\kappa_{0}^{+}(t)=$ $\delta(t), \kappa_{0}^{-}(t)=1+i \cot \pi t, a_{0}^{+}(t, s)=a(t), a_{0}^{-}(t, s)=b(t, s)$. Moreover, $\hat{\kappa}_{0}^{+}(m)=1$, 
$m \in \mathbb{Z}, \hat{\kappa}_{0}^{-}(m)=\operatorname{sign}(m), m \neq 0, \hat{\kappa}_{0}^{-}(0)=1$. Hence $\alpha=0$ and we can choose the parameter $\beta$ to be an arbitrarily large positive number.

As in the above examples, the boundary integral equations usually have nonpositive order, which in terms of $\alpha$ means that $\alpha \geq 0$, but, for example, for a hypersingular integral equation we have $\alpha=-1$. Therefore we allow $\alpha \in \mathbb{R}$ to be arbitrary in (4), (5). The integrals in (2) can be interpreted on the basis of the equalities (which follow from (3))

$$
\begin{gathered}
\int_{0}^{1} \kappa_{0}^{ \pm}(t-s) e^{i m 2 \pi s} d s=\hat{\kappa}_{0}^{ \pm}(m) e^{i m 2 \pi t}, \quad m \in \mathbb{Z}, \\
\int_{0}^{1} \kappa_{p}(t-s) e^{i m 2 \pi s} d s=\hat{\kappa}_{p}(m) e^{i m 2 \pi t}, \quad m \in \mathbb{Z}, p=1, \ldots, q,
\end{gathered}
$$

and the Fourier expansions of $u(s), a^{ \pm}(t, s)$, and $a_{p}(t, s)$. Equivalently, for a smooth $u$ we may interpret the integrals in (2) as duality products between elements of appropriate Sobolev spaces $H^{-\lambda}$ and $H^{\lambda}$, e.g. for $p=1, \ldots, q$

$$
\int_{0}^{1} \kappa_{p}(t-s) a_{p}(t, s) u(s) d s=\left\langle\kappa_{p}(t-s), a_{p}(t, s) u(s)\right\rangle .
$$

The Sobolev space $H^{\lambda}, \lambda \in \mathbb{R}$, consists of 1-periodic functions (distributions) $u$ satisfying

$$
\|u\|_{\lambda}:=\left(\sum_{m \in \mathbb{Z}} \underline{m}^{2 \lambda}|\hat{u}(m)|^{2}\right)^{1 / 2}<\infty
$$

where

$$
\underline{m}=\left\{\begin{array}{ll}
|m|, & m \neq 0 \\
1, & m=0
\end{array}\right\}, \quad m \in \mathbb{Z}
$$

It follows from the inequalities $\left|\kappa_{0}^{ \pm}(m)\right| \leq c_{2}|m|^{-\alpha}, m \in \mathbb{Z}, m \neq 0$ (cf.(4)), that the operator $A_{0}$ is bounded from any $H^{\lambda}$ to $H^{\lambda+\alpha}, \lambda \in \mathbb{R}$ (see Section 2). Similarly, duae to (5), the operator $A_{p}, 1 \leq p \leq q$, is bounded from $H^{\lambda}$ to $H^{\lambda+\alpha+\beta}$ and compact from $H^{\lambda}$ to $H^{\lambda+\alpha}, \lambda \in \mathbb{R}$.

Proposition 1.1. Under conditions (4)-(6), $\mathcal{A}=\sum_{p=0}^{q} A_{p} \in \mathcal{L}\left(H^{\lambda}, H^{\lambda+\alpha}\right)$ is a Fredholm operator of index 0 for any $\lambda \in \mathbb{R}$. Then

$$
\mathcal{N}(\mathcal{A}):=\left\{u \in H^{\lambda}: \mathcal{A} u=0\right\} \subset \bigcap_{\mu \in \mathbb{R}} H^{\mu}=: C_{1}^{\infty} .
$$

A proof follows from considerations of Section 3. Consequently, assuming (4) (6) and

$$
v \in C_{1}^{\infty}, \quad \mathcal{A} v=0 \Rightarrow v=0,
$$

a (bounded) inverse $\mathcal{A}^{-1} \in \mathcal{L}\left(H^{\lambda+\alpha}, H^{\lambda}\right)$ exists for all $\lambda \in \mathbb{R}$.

Our purpose is to construct fast solvers of the optimal convergence order for the problem (1). Introduce the $N$-dimensional space of 1-periodic trigonometric functions

$$
\mathcal{T}_{N}=\left\{v_{N}: v_{N}=\sum_{l \in \mathbb{Z}_{N}} c_{l} e^{i l 2 \pi t}, c_{l} \in \mathbb{C}\right\},
$$


where

$$
\mathbb{Z}_{N}=\left\{l \in \mathbb{Z}:-\frac{N}{2}<l \leq \frac{N}{2}\right\} .
$$

Assuming $f \in H^{\mu+\alpha}, \mu+\alpha>\frac{1}{2}$, we construct in $\mathcal{O}(N \log N)$ arithmetical operations approximations $u_{N} \in \mathcal{T}_{N}$ to $u=\mathcal{A}^{-1} f \in H^{\mu}$ such that

$$
\left\|u_{N}-u\right\|_{\lambda} \leq c_{\lambda, \mu} N^{\lambda-\mu}\|u\|_{\mu}, \quad-\alpha \leq \lambda \leq \mu .
$$

For more special equations, a similar problem setting has been examined by Amosov [1]. His approach is based on the construction of high order parametrices $\mathcal{B}$ for $\mathcal{A}$, i.e. operators $\mathcal{B} \in \mathcal{L}\left(H^{\lambda+\alpha}, H^{\lambda}\right)$ such that $T=I-\mathcal{B} \mathcal{A} \in \mathcal{L}\left(H^{\lambda}, H^{\lambda+\gamma}\right)$ with a sufficiently large $\gamma(\gamma \geq 2(\mu+\alpha))$. The preconditioned equation $\mathcal{B} \mathcal{A} u=\mathcal{B} f$, or $u=T u+\mathcal{B} f$, was used to approximate $\hat{u}(m)$ for large $m$ by $(\widehat{\mathcal{B f}})(m)$; for smaller $m$ the approximations to $\hat{u}(m)$ were found by trigonometric collocation method without use of the preconditioning. A practical restriction of the algorithm is caused by difficulties constructing a parametrix of a sufficiently high order $\gamma$. In our case, without further assumptions about the problem (1), the construction fails. Therefore we make use of a simple $\mathcal{B}$ such that $T=I-\mathcal{B} \mathcal{A} \in \mathcal{L}\left(H^{\lambda}, H^{\lambda+\beta}\right)$. The preconditioned equation $\mathcal{B} \mathcal{A} u=\mathcal{B} f$ will be solved by a special version of a (fully discrete) Galerkin method in a smaller dimension $n(n<N)$, and a simple refinement of the Galerkin solution is used to obtain $u_{N} \in \mathcal{T}_{N}$ of accuracy (8). The matrix form of the method has a band structure compensating the modest smoothing properties of $T$ and keeping the computations in $\mathcal{O}(N \log N)$ arithmetical operations. Compared with a direct solution of the system, the properties of the algorithm will be improved, involving a two grid iteration method to solve the Galerkin equation.

An interesting question arises: Can similar results be obtained when problem (1) is solved directly, without a preconditioning, by a fully discrete Galerkin or collocation method? Recently, McLean, Prössdorf, and Wendland (see[12]-[14]) obtained nice results concerning the trigonometric Galerkin and collocation methods for a certain class of pseudodifferential equations. We also refer to [7], [20], where fully discretized methods are examined for problem (1). It seems possible to reorganize these methods so that the accuracy (8) will be obtained in $\mathcal{O}(N \log N)$ arithmetical operations, but we cannot go into details in this paper. In a rather special case of the Symm's integral equation this was achieved in [19]; the case is covered also by [1] with the simplest preconditioner $\mathcal{B}=A_{0}^{-1}$. More generally, if the coefficients $a_{0}^{ \pm}$ of the main part $A_{0}$ of $\mathcal{A}$ are constant, then it does not matter whether we apply the (trigonometric) Galerkin method directly to (1) or to the equation $\mathcal{B A}=\mathcal{B} f$ with $\mathcal{B}=A_{0}^{-1}$, which is exactly the preconditioner we use in this case (cf. (16)). So our results cover also the (fully discretized) Galerkin method applied directly to (1), provided that $a_{0}^{ \pm}$are constant. For the collocation method, a similar relation is not true, and more sophisticated modifications are needed (see [1]). More specifically, in [17] a method of computational complexity $\mathcal{O}\left(N^{2}\right)$ using collocation is proposed.

Our results are based on a superconvergence property of the Galerkin method (see (59)). Note that this property does not hold if the Galerkin method is applied directly to (1) with non-constant $a_{0}^{ \pm}(t, t)$, without a suitable preconditioning.

Remark 1.1. In some cases where all the functions are analytic, or have isolated singularities, the convergence rate is even exponential. For studies in this direction, see, e.g., Bialecki [2], Saranen and Vainikko [20], and Stenger [21]. 


\section{Integral operators in Sobolev Spaces}

The Sobolev space $H^{\lambda, \mu}, \lambda, \mu \in \mathbb{R}$, consists of 1-biperiodic functions (distributions) $v(t, s)$ satisfying

$$
\|v\|_{\lambda, \mu}:=\left(\sum_{l, m \in \mathbb{Z}} \underline{l}^{2 \lambda} \underline{\underline{m}}^{2 \mu}|\hat{v}(l, m)|^{2}\right)^{1 / 2}<\infty
$$

where

$$
\hat{v}(l, m)=\int_{0}^{1} \int_{0}^{1} v(t, s) e^{-i l 2 \pi t} e^{-i m 2 \pi s} d t d s, \quad l, m \in \mathbb{Z} .
$$

Lemma 2.1 ([7], [20]). Assume that $a(t, s)$ is $C^{\infty}$-smooth and 1-biperiodic, and

$$
|\hat{\kappa}(l)| \leq c|l|^{-\alpha}, \quad l \in \mathbb{Z}, l \neq 0 .
$$

Then the operator $A$ defined by

$$
(A u)(t)=\int_{0}^{1} \kappa(t-s) a(t, s) u(s) d s
$$

is bounded from any $H^{\lambda}$ to $H^{\lambda+\alpha}, \lambda \in \mathbb{R}$, and the following estimates hold for the norm:

(i) if $\alpha \geq 0$ then for all $\lambda \in \mathbb{R}$, with any $\nu>1 / 2$,

$$
\|A\|_{\lambda, \lambda+\alpha}:=\|A\|_{\mathcal{L}\left(H^{\lambda}, H^{\lambda+\alpha}\right)} \leq c_{\lambda, \alpha, \nu}\|a\|_{\max (|\lambda+\alpha|, \nu), \max (|\lambda|, \nu)} ;
$$

(ii) if $\alpha<0$ then (11) remains true for $\lambda \leq 0$ and for $\lambda \geq-\alpha$, whereas for $0<\lambda<-\alpha$, again with an arbitrary $\nu>1 / 2$,

$$
\|A\|_{\lambda, \lambda+\alpha} \leq c_{\lambda, \alpha, \nu} \min \left(\|a\|_{|\lambda+\alpha|+\nu, \max (\lambda, \nu)},\|a\|_{\max (|\lambda+\alpha|, \nu), \lambda+\nu}\right) .
$$

Counter-examples show that (11) may be violated for $0<\lambda<-\alpha, \alpha<0$.

Consequence 2.1 ([7], [20]). If

$$
|\hat{\kappa}(l)-\hat{\kappa}(l-1)| \leq c|l|^{-\alpha-\beta}, \quad l \in \mathbb{Z}, l \neq 0,
$$

and $a(t, t)=0$ for all $t \in \mathbb{R}$, then $A \in \mathcal{L}\left(H^{\lambda}, H^{\lambda+\alpha+\beta}\right), \lambda \in \mathbb{R}$.

Proof. We represent $A$ in the form $(A u)(t)=\int_{0}^{1} \kappa_{1}(t-s) a_{1}(t, s) u(s) d s$, where $\kappa_{1}(t)=\kappa(t)\left(1-e^{i 2 \pi t}\right), a_{1}(t, s)=a(t, s) /\left(1-e^{i 2 \pi(t-s)}\right)$. Since $a$ vanishes on the diagonal, $a_{1}(t, s)$ is $C^{\infty}$-smooth and 1-biperiodic. Since $\hat{\kappa}_{1}(l)=\hat{\kappa}(l)-\hat{\kappa}(l-1)$, we have $\left|\hat{\kappa}_{1}(l)\right| \leq c|l|^{-\alpha-\beta}$; see (13). Now the assertion follows from Lemma 2.1.

The Fourier representation of the operator $A$ defined by (10) is given by

$$
(A u)(t)=\sum_{k \in \mathbb{Z}} \sum_{j \in \mathbb{Z}} \sum_{m \in \mathbb{Z}} \hat{a}(k-m, m-j) \hat{\kappa}(m) \hat{u}(j) e^{i k 2 \pi t} .
$$

Lemma 2.2. If supp $\hat{a}$ is finite then the matrix

$$
\mathbf{A}=\left(\mathbf{a}_{k j}\right)_{k, j \in \mathbb{Z}}, \quad \mathbf{a}_{k j}=\sum_{m \in \mathbb{Z}} \hat{a}(k-m, m-j) \hat{\kappa}(m),
$$

has a band structure. More precisely, if

$$
\operatorname{supp} \hat{a} \subset\left\{\left(k_{1}, k_{2}\right) \in \mathbb{Z}^{2}:\left|k_{1}\right|+\left|k_{2}\right| \leq \frac{r}{2}\right\}, \quad r \in \mathbb{N},
$$

then $\mathbf{a}_{k j}=0$ for $|k-j|>\frac{r}{2}$, and consequently $A \mathcal{T}_{n} \subset \mathcal{T}_{n+r}$. 
Proof. If $\mathbf{a}_{k j} \neq 0$, then $\hat{a}(k-m, m-j) \neq 0$ for a $m \in \mathbb{Z}$, i.e. $|k-m|+|m-j| \leq r / 2$, implying $|k-j| \leq r / 2$. For $u_{n} \in \mathcal{T}_{n}$ we have

$$
\left(\widehat{A u}_{n}\right)(k)=\sum_{j \in \mathbb{Z}_{n}} \mathbf{a}_{k j} \hat{u}_{n}(j), \quad k \in \mathbb{Z} .
$$

For $k \notin \mathbb{Z}_{n+r}, j \in \mathbb{Z}_{n}$ we have $|k-j|>\frac{r}{2}, \mathbf{a}_{k j}=0$. Thus, $\left(\widehat{A u}_{n}\right)(k)=0$ for $k \notin \mathbb{Z}_{n+r}$, i.e. $A u_{n} \in \mathcal{T}_{n+r}$.

\section{Preconditioning of the PRoblem}

Let us return to the problem (1). We shall precondition it by the operator

$$
\mathcal{B}=\left[\left(1 / b_{1}\right) P^{+}+\left(1 / b_{2}\right) P^{-}\right] \Lambda^{-1},
$$

where $b_{1}$ and $b_{2}$ are nonvanishing functions defined in (6) and

$$
\begin{gathered}
P^{+} u=\sum_{l \geq 0} \hat{u}(l) e^{i l 2 \pi t}, \quad P^{-} u=\sum_{l<0} \hat{u}(l) e^{i l 2 \pi t}, \\
\Lambda u=\hat{u}(0)+\sum_{l \in \mathbb{Z}, l \neq 0} \hat{\kappa}_{0}^{+}(l) \hat{u}(l) e^{i l 2 \pi t}, \quad \Lambda^{-1} v=\hat{v}(0)+\sum_{l \in \mathbb{Z}, l \neq 0}\left[\hat{\kappa}_{0}^{+}(l)\right]^{-1} \hat{v}(l) e^{i l 2 \pi t} .
\end{gathered}
$$

Due to the first inequality (4), $\Lambda \in \mathcal{L}\left(H^{\lambda}, H^{\lambda+\alpha}\right), \Lambda^{-1} \in \mathcal{L}\left(H^{\lambda+\alpha}, H^{\lambda}\right)$, and $\mathcal{B} \in$ $\mathcal{L}\left(H^{\lambda+\alpha}, H^{\lambda}\right)$ for any $\lambda \in \mathbb{R}$.

Lemma 3.1. Under conditions (4)-(6) we have, for $\mathcal{A}=\sum_{p=0}^{q} A_{p}$ defined by (2) and $\mathcal{B}$ defined by $(16)$,

$$
T:=I-\mathcal{B A} \in \mathcal{L}\left(H^{\lambda}, H^{\lambda+\beta}\right) \quad \forall \lambda \in \mathbb{R} .
$$

Proof. According to Lemma 2.1 and condition (5), $A_{p} \in \mathcal{L}\left(H^{\lambda}, H^{\lambda+\alpha+\beta}\right), p=$ $1, \ldots, q$; therefore $\mathcal{B} A_{p} \in \mathcal{L}\left(H^{\lambda}, H^{\lambda+\beta}\right), p=1, \ldots, q$. Further, we represent $A_{0}$ in the form $A_{0}=A_{00}+A_{01}$, where

$$
\begin{gathered}
\left(A_{00} u\right)(t)=\int_{0}^{1}\left[\kappa_{0}^{+}(t-s) a_{0}^{+}(s, s)+\kappa_{0}^{-}(t-s) a_{0}^{-}(s, s)\right] u(s) d s \\
=\Lambda\left(P^{+} b_{1} u+P^{-} b_{2} u\right)+\left[\hat{\kappa}_{0}^{+}(0)-1\right] \int_{0}^{1} a_{0}^{+}(s, s) u(s) d s \\
\quad+\left[\hat{\kappa}_{0}^{-}(0)-1\right] \int_{0}^{1} a_{0}^{-}(s, s) u(s) d s, \\
\left(A_{01} u\right)(t)=\int_{0}^{1} \kappa_{0}^{+}(t-s)\left[a_{0}^{+}(t, s)-a_{0}^{+}(s, s)\right] u(s) d s \\
\quad+\int_{0}^{1} \kappa_{0}^{-}(t-s)\left[a_{0}^{-}(t, s)-a_{0}^{-}(s, s)\right] u(s) d s .
\end{gathered}
$$

The function $a_{0}^{ \pm}(t, s)-a_{0}^{ \pm}(s, s)$ vanishes on the diagonal $s=t$, and Consequence 2.1 says that $A_{01} \in \mathcal{L}\left(H^{\lambda}, H^{\lambda+\alpha+\beta}\right), \mathcal{B} A_{01} \in \mathcal{L}\left(H^{\lambda}, H^{\lambda+\beta}\right)$. Finally,

$$
\begin{aligned}
\mathcal{B} \Lambda\left(P^{+} b_{1} u+P^{-} b_{2} u\right) & =\left(1 / b_{1}\right) P^{+} b_{1} u+\left(1 / b_{2}\right) P^{-} b_{2} u \\
& =u+\left(1 / b_{1}\right)\left(P^{+} b_{1}-b_{1} P^{+}\right) u+\left(1 / b_{2}\right)\left(P^{-} b_{2}-b_{2} P^{-}\right) u .
\end{aligned}
$$

To obtain (17), it now suffices to show that, for any $b \in C_{1}^{\infty}$,

$$
P^{ \pm} b-b P^{ \pm} \in \mathcal{L}\left(H^{\lambda}, H^{\mu}\right) \quad \forall \lambda, \mu \in \mathbb{R}
$$


or equivalently,

$$
P^{ \pm} b-b P^{ \pm} \in \mathcal{L}\left(H^{-\lambda}, H^{\lambda}\right) \quad \forall \lambda \geq 0 .
$$

Let us prove (18) for $P^{+}$. We have

$$
P^{+}(b u)=\sum_{l \geq 0}\left[\sum_{k \in \mathbb{Z}} \hat{b}(l-k) \hat{u}(k)\right] e^{i l 2 \pi t}, \quad b P^{+} u=\sum_{l \in \mathbb{Z}}\left\{\sum_{k \geq 0} \hat{b}(l-k) \hat{u}(k)\right\} e^{i l 2 \pi t},
$$

and

$$
\begin{gathered}
P^{+}(b u)-b P^{+} u=\sum_{l \geq 0}\left[\sum_{k<0} \hat{b}(l-k) \hat{u}(k)\right] e^{i l 2 \pi t}-\sum_{l<0}\left[\sum_{k \geq 0} \hat{b}(l-k) \hat{u}(k)\right] e^{i l 2 \pi t}, \\
\left\|P^{+}(b u)-b P^{+} u\right\|_{\lambda}=\left\{\sum_{l \geq 0}\left|\sum_{k<0} \underline{l}^{\lambda} \hat{b}(l-k) \hat{u}(k)\right|^{2}+\left.\left.\sum_{l<0}\left|\sum_{k \geq 0}\right| l\right|^{\lambda} \hat{b}(l-k) \hat{u}(k)\right|^{2}\right\}^{1 / 2} .
\end{gathered}
$$

In the first sum, $l-k>l \geq 0$ and $\frac{l-k}{|k|}=\frac{l+|k|}{|k|} \geq 1$; therefore $\underline{l}^{\lambda} \leq(l-k)^{\lambda} \leq$ $(l-k)^{2 \lambda}|k|^{-\lambda}$. In the second sum, $l-k \leq l<0$ and $\frac{|l-k|}{\underline{k}}=\frac{|l|+k}{\underline{k}} \geq 1$; therefore $|l|^{\lambda} \leq|l-k|^{\lambda} \leq|l-k|^{2 \lambda} \underline{k}^{-\lambda}$. Thus

$$
\left\|P^{+}(b u)-b P^{+} u\right\|_{\lambda} \leq\left\{\sum_{l \in \mathbb{Z}}\left[\sum_{k \in \mathbb{Z}}|l-k|^{2 \lambda} \underline{k}^{-\lambda}|\hat{b}(l-k) \| \hat{u}(k)|\right]^{2}\right\}^{1 / 2}=\|a v\|_{0},
$$

where for given $b$ and $u$ the functions $a$ and $v$ are defined through their Fourier coefficients $\hat{a}(l)=|l|^{2 \lambda}|\hat{b}(l)|, \hat{v}(k)=\underline{k}^{-\lambda}|\hat{u}(k)|, k, l \in \mathbb{Z}$. Further,

$$
\|a v\|_{0} \leq \max _{t}|a(t)|\|v\|_{0} \leq \sum_{l}|\hat{a}(l)|\|u\|_{-\lambda} \leq \gamma_{\nu}\|b\|_{2 \lambda+\nu}\|u\|_{-\lambda},
$$

where

$$
\gamma_{\nu}=\left(\sum_{l \in \mathbb{Z}} \underline{l}^{-2 \nu}\right)^{1 / 2}=\left(1+2 \sum_{l=1}^{\infty} l^{-2 \nu}\right)^{1 / 2}<\infty, \quad \nu>\frac{1}{2}
$$

We get

$$
\left\|P^{+}(b u)-b P^{+} u\right\|_{\lambda} \leq \gamma_{\nu}\|b\|_{2 \lambda+\nu}\|u\|_{-\lambda}, \quad \lambda \geq 0,
$$

proving (18) for $P^{+}$. For $P^{-}$the proof is similar.

Lemma 3.2. Under conditions (4), (6) the operator $\mathcal{B} \in \mathcal{L}\left(H^{\lambda+\alpha}, H^{\lambda}\right)$ has a bounded inverse $\mathcal{B}^{-1} \in \mathcal{L}\left(H^{\lambda}, H^{\lambda+\alpha}\right)$ for any $\lambda \in \mathbb{R}$.

Proof. It is well known (see e.g. [4] or [15]) that (6) is a necessary and sufficient condition for the existence of bounded inverses to $B_{1}=b_{1} P^{+}+b_{2} P^{-}$and $B_{2}=$ $\left(1 / b_{1}\right) P^{+}+\left(1 / b_{2}\right) P^{-}$considered as operators in $H^{0}=L^{2}(0,1)$. Considering $B_{1}$ and $B_{2}$ as operators in $H^{\lambda}$, we have (see (18))

$$
B_{1} B_{2}=I-S_{1}, \quad B_{2} B_{1}=I-S_{2},
$$

where $S_{1}, S_{2} \in \mathcal{L}\left(H^{\lambda}, H^{\mu}\right)$ with any $\mu>\lambda$. We see that

$$
\mathcal{N}\left(B_{1}\right) \subset \mathcal{N}\left(I-S_{2}\right) \subset C_{1}^{\infty} \subset H^{0},
$$


and consequently $\mathcal{N}\left(B_{1}\right)=\{0\}$. Similarly, $\mathcal{N}\left(B_{2}\right)=\{0\}$, implying also $\mathcal{N}\left(I-S_{1}\right)=$ $\mathcal{N}\left(I-S_{2}\right)=\{0\}$. Since

$$
B_{2} H^{\lambda} \supset B_{2} B_{1} H^{\lambda}=\left(I-S_{2}\right) H^{\lambda}=H^{\lambda},
$$

the operator $B_{2} \in \mathcal{L}\left(H^{\lambda}, H^{\lambda}\right)$ has a bounded inverse. This together with the properties of $\Lambda$ mentioned above proves the lemma.

Proof of Proposition 1.1. Assume (4) - (6). Due to Lemmas 3.1 and 3.2 we can represent $\mathcal{A}=\mathcal{B}^{-1}(I-T)$, and the Fredholm properties of $A \in \mathcal{L}\left(H^{\lambda}, H^{\lambda+\alpha}\right)$ follow from the compactness of $T \in \mathcal{L}\left(H^{\lambda}, H^{\lambda}\right)$. Further, if $\mathcal{A} v=0$ for a $v \in H^{\lambda}$, then $\mathcal{B} \mathcal{A} v=0, v=T v, v=T^{n} v, n=1,2, \ldots$, and $v \in H^{\mu}$ with any $\mu \in \mathbb{R}$, due to the smoothing property (17) of $T$.

According to Lemmas 3.1 and 3.2, problem (1) is equivalent to the problem

$$
\mathcal{B A} u=\mathcal{B} f
$$

as well as to the problem

$$
u=T u+g, \quad g=\mathcal{B} f, \quad T=I-\mathcal{B A} \in \mathcal{L}\left(H^{\lambda}, H^{\lambda+\beta}\right) .
$$

We shall use the formulation (19), constructing a discretization of (1) and (20) to examine the convergence properties of it.

\section{TRIgONOMETRIC INTERPOLATION}

Here we present some technical tools for discretization of the problem. The Fourier projection $P_{n} u$ of $u \in H^{\mu}, \mu \in \mathbb{R}$, is defined by

$$
P_{n} u=\sum_{k \in \mathbb{Z}_{n}} \hat{u}(k) e^{i k 2 \pi t} \in \mathcal{T}_{n}, \quad n \in \mathbb{N}
$$

(see the notations of Section 1). Obviously, $P_{n}$ is an orthogonal projection operator in any $H^{\mu}, \mu \in \mathbb{R}$. It is clear also that

$$
\left\|u-P_{n} u\right\|_{\lambda} \leq(n / 2)^{\lambda-\mu}\|u\|_{\mu}, \quad \lambda \leq \mu .
$$

The interpolation projection $Q_{n} u$ is defined by the conditions

$$
Q_{n} u \in \mathcal{T}_{n}, \quad\left(Q_{n} u\right)\left(j n^{-1}\right)=u\left(j n^{-1}\right), \quad j=1, \ldots, n,
$$

for $u \in H^{\mu}, \mu>\frac{1}{2}$, guaranteeing the continuity of $u$. It is known (see [1], [18]) that

$$
\left\|u-Q_{n} u\right\|_{\lambda} \leq c_{\lambda, \mu} n^{\lambda-\mu}\|u\|_{\mu}, \quad 0 \leq \lambda \leq \mu, \mu>\frac{1}{2} .
$$

Preparing for the two dimensional case, we first specify the constant $c_{\lambda, \mu}$.

Lemma 4.1. For $u \in H^{\mu}, \mu>\frac{1}{2}$, we have

$$
\left\|u-Q_{n} u\right\|_{\lambda} \leq \gamma_{\mu}(n / 2)^{\lambda-\mu}\|u\|_{\mu}, \quad 0 \leq \lambda \leq \mu,
$$

where

$$
\gamma_{\mu}=\left(1+2 \sum_{j=1}^{\infty} \frac{1}{j^{2 \mu}}\right)^{1 / 2}<\infty, \quad \mu>\frac{1}{2}
$$


Proof. Due to (21) and the equality

$$
\left\|u-Q_{n} u\right\|_{\lambda}^{2}=\left\|P_{n} u-Q_{n} u\right\|_{\lambda}^{2}+\left\|\left(I-P_{n}\right) u\right\|_{\lambda}^{2}
$$

we have to estimate the norm $\left\|P_{n} u-Q_{n} u\right\|^{2}$. It is easy to see that

$$
\left(Q_{n} \widehat{u-P_{n}} u\right)(k)= \begin{cases}\sum_{0 \neq j \in \mathbb{Z}} \hat{u}(k+j n), & k \in \mathbb{Z}_{n}, \\ 0, & k \in \mathbb{Z} \backslash \mathbb{Z}_{n}\end{cases}
$$

therefore

$$
\begin{gathered}
\left\|Q_{n} u-P_{n} u\right\|_{\lambda}^{2}=\sum_{k \in \mathbb{Z}_{n}} \underline{k}^{2 \lambda}\left|\sum_{0 \neq j \in \mathbb{Z}} \hat{u}(k+j n)\right|^{2} \\
=\sum_{k \in \mathbb{Z}_{n}}\left[\sum_{0 \neq j \in \mathbb{Z}} \frac{\underline{k}^{\lambda}}{|k+j n|^{\mu}} \cdot|k+j n|^{\mu}|\hat{u}(k+j n)|\right]^{2} \leq \epsilon_{n}^{2}\|u\|_{\mu}^{2},
\end{gathered}
$$

where

$$
\epsilon_{n}^{2}=\max _{k \in \mathbb{Z}} \sum_{0 \neq j \in \mathbb{Z}} \frac{\underline{k}^{2 \lambda}}{|k+j n|^{2 \mu}} \leq \max \left(n^{-2 \mu} \sum_{0 \neq j \in \mathbb{Z}} \frac{1}{|j|^{2 \mu}}, \max _{1 \leq x \leq n / 2} \sum_{0 \neq j \in \mathbb{Z}} \frac{x^{2 \lambda}}{|x+j n|^{2 \mu}}\right) .
$$

The function

$$
x \longrightarrow \frac{x^{2 \lambda}}{|x+j n|^{2 \mu}}+\frac{x^{2 \lambda}}{|x-j n|^{2 \mu}}=x^{2 \lambda}\left(\frac{1}{(j n+x)^{2 \mu}}+\frac{1}{(j n-x)^{2 \mu}}\right), \quad j \in \mathbb{N},
$$

increases on $[1, n / 2]$; therefore the maximum is attained at $x=n / 2$, and

$$
\begin{aligned}
\max _{1 \leq x \leq n / 2} \sum_{0 \neq j \in \mathbb{Z}} \frac{x^{2 \lambda}}{|x+j n|^{2 \mu}}= & \left(\frac{n}{2}\right)^{2(\lambda-\mu)} \sum_{j=1}^{\infty}\left[\frac{1}{(2 j+1)^{2 \mu}}+\frac{1}{(2 j-1)^{2 \mu}}\right] \\
& \leq\left(\frac{n}{2}\right)^{2(\lambda-\mu)} 2 \sum_{j=1}^{\infty} \frac{1}{j^{2 \mu}} .
\end{aligned}
$$

Thus

$$
\begin{gathered}
\epsilon_{n}^{2} \leq\left(\frac{n}{2}\right)^{2(\lambda-\mu)} 2 \sum_{j=1}^{\infty} \frac{1}{j^{2 \mu}}, \\
\left\|Q_{n} u-P_{n} u\right\|_{\lambda}^{2} \leq\left(\frac{n}{2}\right)^{2(\lambda-\mu)} 2 \sum_{j=1}^{\infty} \frac{1}{j^{2 \mu}}\|u\|_{\mu}^{2}, \quad 0 \leq \lambda \leq \mu, \mu>1 / 2,
\end{gathered}
$$

and together with (21) this yields (22), (23).

For $v \in H^{\mu_{1}, \mu_{2}}$ and $n_{1}, n_{2} \in \mathbb{N}$, the two dimensional projections $P_{n_{1}, n_{2}} v$ and $Q_{n_{1}, n_{2}} v$ are defined by

$$
\begin{gathered}
P_{n_{1}, n_{2}} v=\sum_{k_{1} \in \mathbb{Z}_{n_{1}}} \sum_{k_{2} \in \mathbb{Z}_{n_{2}}} \hat{v}\left(k_{1}, k_{2}\right) e^{i k_{1} 2 \pi t} e^{i k_{2} 2 \pi s} \in \mathcal{T}_{n_{1}} \otimes \mathcal{T}_{n_{2}}, \\
Q_{n_{1}, n_{2}} v \in \mathcal{T}_{n_{1}} \otimes \mathcal{T}_{n_{2}}, \quad\left(Q_{n_{1}, n_{2}} v\right)\left(j_{1} n_{1}^{-1}, j_{2} n_{2}^{-1}\right)=v\left(j_{1} n_{1}^{-1}, j_{2} n_{2}^{-1}\right), \\
j_{1}=1, \ldots, n_{1}, \quad j_{2}=1, \ldots, n_{2} .
\end{gathered}
$$


Obviously

$$
\begin{gathered}
\left\|v-P_{n_{1}, n_{2}} v\right\|_{\lambda_{1}, \lambda_{2}} \leq \max \left[\left(\frac{n_{1}}{2}\right)^{\lambda_{1}-\mu_{1}},\left(\frac{n_{2}}{2}\right)^{\lambda_{2}-\mu_{2}}\right]\|v\|_{\mu_{1}, \mu_{2}}, \\
\lambda_{1} \leq \mu_{1}, \lambda_{2} \leq \mu_{2} .
\end{gathered}
$$

Lemma 4.2. For $v \in H^{\mu_{1}, \mu_{2}} \mu_{1}>\frac{1}{2}$, and $\mu_{2}>\frac{1}{2}$,

$$
\begin{aligned}
\left\|v-Q_{n_{1}, n_{2}} v\right\|_{\lambda_{1}, \lambda_{2}} & \leq\left[\gamma_{\mu_{1}}\left(\frac{n_{1}}{2}\right)^{\lambda_{1}-\mu_{1}}+\gamma_{\mu_{2}}\left(\frac{n_{2}}{2}\right)^{\lambda_{2}-\mu_{2}}\right]\|v\|_{\mu_{1}, \mu_{2}}, \\
0 & \leq \lambda_{1} \leq \mu_{1}, 0 \leq \lambda_{2} \leq \mu_{2},
\end{aligned}
$$

where $\gamma_{\mu_{1}}$ and $\gamma_{\mu_{2}}$ are defined by (23).

Proof. This time we have (cf. the proof of Lemma 4.1)

$$
\left\|v-Q_{n_{1}, n_{2}} v\right\|_{\lambda_{1}, \lambda_{2}}^{2}=\left\|P_{n_{1}, n_{2}} v-Q_{n_{1}, n_{2}} v\right\|_{\lambda_{1}, \lambda_{2}}^{2}+\left\|v-P_{n_{1}, n_{2}} v\right\|_{\lambda_{1}, \lambda_{2}}^{2}
$$

with

$$
\begin{gathered}
\left(Q_{n_{1}, n_{2}} \widehat{\widehat{v-P}}{ }_{n_{1}, n_{2}} v\right)\left(k_{1}, k_{2}\right) \\
= \begin{cases}\sum_{\left|j_{1}\right|+\left|j_{2}\right| \geq 1} \hat{v}\left(k_{1}+j_{1} n_{1}, k_{2}+j_{2} n_{2}\right), & \left(k_{1}, k_{2}\right) \in \mathbb{Z}_{n_{1}} \times \mathbb{Z}_{n_{2}}, \\
0, & \text { for other }\left(k_{1}, k_{2}\right),\end{cases} \\
\left\|Q_{n_{1}, n_{2}} v-P_{n_{1}, n_{2}} v\right\|_{\lambda_{1}, \lambda_{2}}^{2} \leq \epsilon_{n_{1}, n_{2}}^{2}\|v\|_{\mu_{1}, \mu_{2}}^{2},
\end{gathered}
$$

where

$$
\begin{aligned}
\epsilon_{n_{1}, n_{2}}^{2} & =\max _{k_{1} \in \mathbb{Z}_{n_{1}}, k_{2} \in \mathbb{Z}_{n_{2}}} \sum_{j_{1}, j_{2} \in \mathbb{Z},\left|j_{1}\right|+\left|j_{2}\right| \geq 1} \frac{\underline{k}_{1}^{2 \lambda_{1}}}{\left(\underline{k_{1}+j_{1} n_{1}}\right)^{2 \mu_{1}}} \frac{\underline{k}_{2}^{2 \lambda_{2}}}{\left(\underline{k_{2}+j_{2} n_{2}}\right)^{2 \mu_{2}}} \\
& \leq \epsilon_{n_{1}}^{2}+\epsilon_{n_{2}}^{2}+\epsilon_{n_{1}}^{2} \epsilon_{n_{2}}^{2}
\end{aligned}
$$

with

$$
\epsilon_{n_{1}}^{2}=\max _{k_{1} \in \mathbb{Z}_{n_{1}}} \sum_{0 \neq j_{1} \in \mathbb{Z}} \frac{\underline{k}_{1}^{2 \lambda_{1}}}{\left|k_{1}+j_{1} n_{1}\right|^{2 \mu_{1}}}, \quad \epsilon_{n_{2}}^{2}=\max _{k_{2} \in \mathbb{Z}_{n_{2}}} \sum_{0 \neq j_{2} \in \mathbb{Z}} \frac{\underline{k}_{2}^{2 \lambda_{2}}}{\left|k_{2}+j_{2} n_{2}\right|^{2 \mu_{2}}} .
$$

Estimating these numbers as in (24) and making use of (25), we obtain (26). The constant in (26) can be somewhat reduced.

For a set $D \subset \mathbb{Z}^{2}$ and $v \in H^{\mu_{1}, \mu_{2}}$ we denote

$$
P_{D} v=\sum_{\left(k_{1}, k_{2}\right) \in D} \hat{v}\left(k_{1}, k_{2}\right) e^{i k_{1} 2 \pi t} e^{i k_{2} 2 \pi s}
$$

Lemma 4.3. Assume that

$$
\left\{\left(k_{1}, k_{2}\right) \in \mathbb{Z}^{2}: \underline{k}_{1} \underline{k}_{2}<\frac{1}{2}\right\} \subset D_{n} \subset \mathbb{Z}^{2} .
$$

Then for $0 \leq \lambda_{1} \leq \mu_{1}, 0 \leq \lambda_{2} \leq \mu_{2}, \mu_{1}-\lambda_{1}=\mu_{2}-\lambda_{2}, \mu_{1}>\frac{1}{2}, \mu_{2}>\frac{1}{2}, v \in H^{\mu_{1}, \mu_{2}}$ we have

$$
\left\|v-P_{D_{n}} Q_{n, n} v\right\|_{\lambda_{1}, \lambda_{2}} \leq\left(\gamma_{\mu_{1}}+\gamma_{\mu_{2}}\right)\left(\frac{n}{2}\right)^{\lambda_{1}-\mu_{1}}\|v\|_{\mu_{1}, \mu_{2}} .
$$


Proof. Obviously

$$
\left\|v-P_{D_{n}} Q_{n, n} v\right\|_{\lambda_{1}, \lambda_{2}}^{2} \leq\left\|v-Q_{n, n} v\right\|_{\lambda_{1}, \lambda_{2}}^{2}+\left\|\left(I-P_{D_{n}}\right) v\right\|_{\lambda_{1}, \lambda_{2}}^{2} .
$$

Due to (28) we still have

$$
\left\|v-P_{D_{n}} v\right\|_{\lambda_{1}, \lambda_{2}} \leq \max _{\left(k_{1}, k_{2}\right) \in \mathbb{Z}^{2} \backslash D_{n}} \underline{k}_{1}^{\lambda_{1}-\mu_{1}} \underline{k}_{2}^{\lambda_{2}-\mu_{2}}\|v\|_{\mu_{1}, \mu_{2}} \leq\left(\frac{n}{2}\right)^{\lambda_{1}-\mu_{1}}\|v\|_{\mu_{1}, \mu_{2}} .
$$

Now, in a rough formulation, (29) follows from (26). To obtain the constant in the form (29), one has to revisit the argument of the proof of Lemma 4.2, adding $\left\|v-P_{n, n} v\right\|_{\lambda_{1}, \lambda_{2}}^{2}$ to the term with $\epsilon_{n_{1}}^{2}$ and $\left\|v-P_{D_{n}} v\right\|_{\lambda_{1}, \lambda_{2}}^{2}$ to the term with $\epsilon_{n_{2}}^{2}$.

Estimate (29) allows us to drop a greater part of Fourier coefficients of $Q_{n, n} v$ while maintaining the convergence order of the truncated interpolation $P_{D_{n}} Q_{n, n} v$. Even the constants in (29) and (26) are equal (as we remarked, actually the constant in (26) can be somewhat reduced).

\section{Discretization of the Problem AND COMPUTATIONAL COSTS}

From now on, $N, n, l, m$ are natural numbers,

$$
n \sim N^{\rho}, \quad l \sim N^{\sigma}, \quad m \sim N^{\tau}, \quad 0<\rho, \sigma, \tau<1 ;
$$

further conditions on $\rho, \sigma, \tau$ will be added later. The relation $n \sim N^{\rho}$ means that $c_{1} \leq n / N^{\rho} \leq c_{2}$ as $N \rightarrow \infty$, where $0<c_{1} \leq c_{2}<\infty$.

We approximate the operator $\mathcal{A}=\sum_{p=0}^{q} A_{p}$ by the operator $\mathcal{A}_{l}=\sum_{p=0}^{q} A_{p, l}$, where

$$
\begin{aligned}
\left(A_{0, l} u\right)(t) & =\int_{0}^{1}\left[\kappa_{0}^{+}(t-s) a_{0, l}^{+}(t, s)+\kappa_{0}^{-}(t-s) a_{0, l}^{-}(t, s)\right] u(s) d s, \\
\left(A_{p, l} u\right)(t) & =\int_{0}^{1} \kappa_{p}(t-s) a_{p, l}(t, s) u(s) d s, \\
a_{0, l}^{ \pm} & =P_{\Omega_{l}} Q_{l, l} a_{0}^{ \pm}, \quad a_{p, l}=P_{\Omega_{l}} Q_{l, l} a_{p}, \\
\left\{\left(k_{1}, k_{2}\right) \in \mathbb{Z}^{2}: \underline{k}_{1} \underline{k}_{2}<\frac{l}{2}\right\} & \subset \Omega_{l} \subset\left\{\left(k_{1}, k_{2}\right) \in \mathbb{Z}^{2}:\left|k_{1}\right|+\left|k_{2}\right| \leq \frac{l}{2}\right\}
\end{aligned}
$$

(see $(27)$ ). The operator $\mathcal{B}$ (see (16)) we approximate by

$$
\mathcal{B}_{l}=\left(b_{l}^{+} P^{+}+b_{l}^{-} P^{-}\right) \Lambda^{-1}, \quad b_{l}^{+}=Q_{l}\left(1 / b_{1}\right), \quad b_{l}^{-}=Q_{l}\left(1 / b_{2}\right) .
$$

According to (11) we have for $\lambda \leq 0$ and for $\lambda+\alpha \geq 0$

$$
\begin{gathered}
\left\|\mathcal{A}_{l}-\mathcal{A}\right\|_{\mathcal{L}\left(H^{\lambda}, H^{\lambda+\alpha}\right)} \\
\leq c_{\lambda, \nu}\left[\left\|a_{0, l}^{+}-a_{0}^{+}\right\|_{\lambda_{1}, \lambda_{2}}+\left\|a_{0, l}^{-}-a_{0}^{-}\right\|_{\lambda_{1}, \lambda_{2}}+\sum_{p=1}^{q}\left\|a_{p, l}-a_{p}\right\|_{\lambda_{1}, \lambda_{2}}\right], \\
\lambda_{1}=\max (|\lambda+\alpha|, \nu), \quad \lambda_{2}=\max (|\lambda|, \nu), \quad \nu>1 / 2,
\end{gathered}
$$

and Lemma 4.3 together with the relation $l \sim N^{\sigma}$ yields

$$
\left\|\mathcal{A}_{l}-\mathcal{A}\right\|_{\mathcal{L}\left(H^{\lambda}, H^{\lambda+\alpha}\right)} \leq c_{\lambda, r} N^{-r} \quad \forall r>0 .
$$


For $\alpha<0,0<\lambda<-\alpha$ we obtain the same results through (12). Making use of (22), we also obtain

$$
\left\|\mathcal{B}_{l}-\mathcal{B}\right\|_{\mathcal{L}\left(H^{\lambda+\alpha}, H^{\lambda}\right)} \leq c_{\lambda, r} N^{-r} \quad \forall r>0 .
$$

We propose the following method to find an approximate solution $u_{n, N} \in \mathcal{T}_{N}$ of equation (1): determine the solution $u_{n} \in \mathcal{T}_{n}$ of the Galerkin equation (cf. (19))

$$
P_{n} \mathcal{B}_{l} \mathcal{A}_{l} u_{n}=P_{n} g_{l, N}, \quad g_{l, N}=\mathcal{B}_{l} Q_{N} f,
$$

and refine it by the formula

$$
u_{n, N}=u_{n}+\left(P_{N}-P_{n}\right) g_{l, N} .
$$

We also propose to use the following two grid iteration method for solving (35): fix an $m<n$; starting from $u_{n}^{0}=0$ compute $u_{n}^{k} \in \mathcal{T}_{n}, k=1,2, \ldots$, via the iteration formula

$$
\begin{aligned}
v_{n}^{k-1} & =P_{n} \mathcal{B}_{l} \mathcal{A}_{l} u_{n}^{k-1}-P_{n} g_{l, N}, \\
u_{n}^{k} & =u_{n}^{k-1}-\left(I-P_{m}\right) v_{n}^{k-1}-\left(P_{m} \mathcal{B}_{l} \mathcal{A}_{l}\right)^{-1} P_{m} v_{n}^{k-1} .
\end{aligned}
$$

After a final iteration step we refine the result, putting (cf.(36))

$$
u_{n, N}^{k}=u_{n}^{k}+\left(P_{N}-P_{n}\right) g_{l, N} .
$$

Let us comment on the implementation and computational costs of the methods (35), (36) and (37), (38). According to the construction (see (31)) we have

$$
\operatorname{supp} \hat{a}_{o, l}^{ \pm}, \operatorname{supp} \hat{a}_{p, l} \subset\left\{\left(k_{1}, k_{2}\right) \in \mathbb{Z}^{2}:\left|k_{1}\right|+\left|k_{2}\right| \leq \frac{l}{2}\right\}, \quad p=1, \ldots, q .
$$

Consequently (see Lemma 2.2), $\mathcal{A}_{l} \mathcal{T}_{n} \subset \mathcal{T}_{n+l}$, and for $u_{n} \in \mathcal{T}_{n}$,

$$
\left(\widehat{\mathcal{A}_{l} u_{n}}\right)(k)=\sum_{j \in \mathbb{Z}_{n}} \mathbf{a}_{k j} \hat{u}_{n}(j), \quad k \in \mathbb{Z}_{n+l},
$$

where (cf. (15))

$$
\begin{gathered}
\mathbf{a}_{k j}=\sum_{M \in \mathbb{Z}}\left[\hat{a}_{0, l}^{+}(k-M, M-j) \hat{\kappa}_{0}^{+}(M)+\hat{a}_{0, l}^{-}(k-M, M-j) \hat{\kappa}_{0}^{-}(M)\right. \\
\left.+\sum_{p=1}^{q} \hat{a}_{p, l}(k-M, M-j) \hat{\kappa}_{p}(M)\right], \quad|k-j| \leq \frac{l}{2}, \\
\mathbf{a}_{k j}=0, \quad|k-j|>\frac{l}{2} .
\end{gathered}
$$

The Fourier coefficients $\hat{a}_{0, l}^{ \pm}\left(k_{1}, k_{2}\right), \hat{a}_{p l}\left(k_{1}, k_{2}\right),\left|k_{1}\right|+\left|k_{2}\right| \leq l / 2$, coincide with the corresponding Fourier coefficients of $Q_{l, l} a_{0}^{ \pm}, Q_{l, l} a_{p}$. The latter can be found from the grid values $a_{0}^{ \pm}\left(j_{1} l^{-1}, j_{2} l^{-1}\right), a_{p}\left(j_{1} l^{-1}, j_{2} l^{-1}\right), j_{1}, j_{2}=1, \ldots, l$, by FFT in $\mathcal{O}\left(l^{2} \log l\right)=\mathcal{O}\left(N^{2 \sigma} \log N\right)$ arithmetical operations. To keep this within $\mathcal{O}(N \log N)$ we set the condition $\sigma \leq 1 / 2$. Further, for a cheap evaluation of sums over $M$ in (40), one can utilize the convolution structure of those when $k$ and $j$ vary on a fixed diagonal $k-j=$ const. By FFT the entries $\mathbf{a}_{k j}$ on a diagonal $k-j=$ const can be found in $\mathcal{O}((n+l) \log (n+l))$ arithmetical operations, and we have to compute 
$\min (n, l+1)$ diagonals. This adds up to $\mathcal{O}\left(N^{\rho+\sigma} \log N\right)$ arithmetical operations. The conditions

$$
\sigma \leq \frac{1}{2}, \quad \rho+\sigma \leq 1
$$

guarantee that the entries $\mathbf{a}_{k j}, k \in \mathbb{Z}_{n+l}, j \in \mathbb{Z}_{n}$, are available in $\mathcal{O}(N \log N)$ arithmetical operations.

If $\sigma \geq \rho$ then $\mathbf{A}_{n}=\left(\mathbf{a}_{k j}\right)_{k \in \mathbb{Z}_{n+l}, j \in \mathbb{Z}_{n}}$ is a full matrix, and an application of $\mathcal{A}_{l}$ to a $u_{n} \in \mathcal{T}_{n}$ by $(39)$ costs, due to $(41),(n+l) n=\mathcal{O}\left(N^{\rho+\sigma}\right)=\mathcal{O}(N)$ multiplications and additions. If $\sigma<\rho$ then $\mathbf{A}_{n}$ is band matrix with band width $l+1$, and an application of $\mathcal{A}_{l}$ to $u_{n} \in \mathcal{T}_{n}$ again costs $(n+l)(l+1)=\mathcal{O}\left(N^{\rho+\sigma}\right)=\mathcal{O}(N)$.

The Fourier coefficients of $Q_{N} f$ can be found by FFT in $\mathcal{O}(N \log N)$ aritmetical operations. After that, using the definitions of $\Lambda^{-1}$ and $P^{ \pm}$(see Section 3), we find in $\mathcal{O}(N)$ arithmetical operations the Fourier coefficients of $P^{ \pm} \Lambda^{-1} Q_{N} f$. Multiplying these polynomials respectively by polynomials $b_{l}^{ \pm}$and adding the results, we obtain $g_{l, N}=\mathcal{B}_{l} Q_{N} f$ (see (32)). To keep the computations in $\mathcal{O}(N \log N)$ operations, the last multiplications must also be performed by FFT. In this way we actually do not need the Fourier coefficients of $b_{l}^{ \pm}$but only the grid values of $1 / b_{1}$ and $1 / b_{2}$. So, the Fourier coefficients of $g_{l, N}$ are available in $\mathcal{O}(N \log N)$ arithmetical operations. Now $P_{n} g_{l, N}$ and $\left(P_{N}-P_{n}\right) g_{l, N}$ occuring in (35)-(38) can be found by simple truncations. A similar scheme can be applied when $\mathcal{B}_{l} v_{n} \in \mathcal{T}_{n+2 l}$ is computed for $v_{n}=\mathcal{A}_{l} u_{n} \in \mathcal{T}_{n+l}, u_{n} \in \mathcal{T}_{n}$, but perhaps it is not the best way.

Let us present an explicit matrix form of an application of $\mathcal{B}_{l}$ to a $v_{n} \in \mathcal{T}_{n+l}$ :

$$
\begin{gathered}
\left(\widehat{\mathcal{B}_{l} v_{n}}\right)(h)=\sum_{k \in \mathbb{Z}_{n+l}} \mathbf{b}_{h k} \hat{v}_{n}(k), \quad h \in \mathbb{Z}_{n+2 l}, \\
\mathbf{b}_{h k}=\left\{\begin{array}{ll}
\hat{b}_{l}^{+}(h-k)\left[\hat{\kappa}_{0}^{+}(k)\right]^{-1}, & k>0 \\
\hat{b}_{l}^{+}(h), & k=0 \\
\hat{b}_{l}^{-}(h-k)\left[\kappa_{0}^{+}(k)\right]^{-1}, & k<0
\end{array}\right\}, \quad h \in \mathbb{Z}_{n+2 l}, k \in \mathbb{Z}_{n+l} .
\end{gathered}
$$

Note that $\operatorname{supp} \hat{b}_{l}^{ \pm} \subset \mathbb{Z}_{l}$. Under conditions (41) the computation of $\mathcal{B}_{l} v_{n}, v_{n} \in \mathcal{T}_{n+l}$, via $(42)$ costs $(n+2 l)(l+1)=\mathcal{O}(N)$ multiplications and additions provided that the Fourier coefficients of $b_{l}^{ \pm}$have been found (what costs only $\mathcal{O}\left(N^{\sigma} \log N\right)$ by FFT).

The matrix representation of $P_{n} B_{l} A_{l} u_{n}, u_{n} \in \mathcal{T}_{n}$, follows from (39), (40) and (42), (43):

$$
\begin{gathered}
\left(P_{n} \widehat{B_{l} A_{l}} u_{n}\right)(h)=\sum_{j \in \mathbb{Z}_{n}} \mathbf{c}_{h j} \hat{u}_{n}(j), \quad h \in \mathbb{Z}_{n}, \\
\mathbf{c}_{h j}=\sum_{k \in \mathbb{Z}_{n+l}} \mathbf{b}_{h k} \mathbf{a}_{k j}, \quad h, j \in \mathbb{Z}_{n} .
\end{gathered}
$$

The matrix form of the Galerkin method (35) reads as follows:

$$
\sum_{j \in \mathbb{Z}_{n}} \mathbf{c}_{h j} \hat{u}_{n}(j)=\hat{g}_{l, N}(h), \quad h \in \mathbb{Z}_{n} .
$$

If $\rho \leq \frac{1}{3}$, then the computation of $\mathbf{C}_{n}=\left(\mathbf{c}_{h j}\right)_{h, j \in \mathbb{Z}_{n}}$ by (45) using FFT costs $\mathcal{O}(n(n+l) \log (n+l)) \leq \mathcal{O}(N)$ and the solving of $(46)$ by the Gauss method costs $\mathcal{O}\left(n^{3}\right) \leq \mathcal{O}(N)$ arithmetical operations. If $\rho \in\left(\frac{1}{3}, 1\right)$ and we impose on $\sigma$ the condition $\rho+2 \sigma \leq 1$ (implying $\sigma<\frac{1}{3}<\rho$ ), then the costs will be again $\mathcal{O}(N)$. 
Indeed, both $\mathbf{A}_{n}$ and $\mathbf{B}_{n}$ are band matrices of band width $l+1$; therefore the computation of $\mathbf{C}_{n} \operatorname{costs} \mathcal{O}\left(n l^{2}\right)=\mathcal{O}\left(N^{\rho+2 \sigma}\right) \leq \mathcal{O}(N)$. The matrix $\mathbf{C}_{n}$ is a band matrix of band width $2 l+1$; therefore the solving of (46) by Gauss method, with pivoting along columns under the main diagonal, also costs $\mathcal{O}\left(n l^{2}\right) \leq \mathcal{O}(N)$. Let us summarize, keeping in mind (41):

Proposition 5.1. If $0<\rho \leq \frac{1}{3}, 0<\sigma \leq \frac{1}{2}$, or if $\frac{1}{3}<\rho<1, \rho+2 \sigma \leq 1$, then method (36) with a direct solution of Galerkin's equation (35) can be implemented in $\mathcal{O}(N \log N)$ arithmetical operations.

We are not free in the choice of $\rho$ so far as we want to obtain an approximation $u_{n, N}$ or $u_{n, N}^{k}$ of an optimal convergence order (see Theorem 6.1, condition (49)). For $\rho$ close to 1 , the condition $\rho+2 \sigma \leq 1$ allows only very small $\sigma>0$. Since (33), (34) hold for any $\sigma>0$, this does not influence the asymptotic properties of the method. To make the method more practical for moderate $N$, we relax the condition $\rho+2 \sigma \leq 1$ by using two grid iterations (37), where the inversions are involved in a smaller dimension $m<n$. In Section 6 we prove that an optimal accuracy of $u_{n, N}^{k}$ defined by (38) is achieved for fixed $k$ which is independent of $N$ (see (51)). Therefore, the amount of arithmetical work is determined by the cost of one iteration step. Instead of (46), we now have to solve similar systems in the dimension $m$ to compute $w_{m}=\left(P_{m} \mathcal{B}_{l} \mathcal{A}_{l}\right)^{-1} P_{m} v_{n}^{k-1} \in \mathcal{T}_{m}$ :

$$
\sum_{j \in \mathbb{Z}_{m}} \mathbf{c}_{h j} \hat{w}_{m}(j)=\hat{v}_{n}^{k-1}(h), \quad h \in \mathbb{Z}_{m},
$$

where

$$
\mathbf{c}_{h j}=\sum_{k \in \mathbb{Z}_{m+l}} \mathbf{b}_{h k} \mathbf{a}_{k j}, \quad h, j \in \mathbb{Z}_{m} .
$$

The analysis of computational costs is similar to the above. Formally, we simply must replace $n \sim N^{\rho}$ by $m \sim N^{\tau}$, i.e. $\rho$ by $\tau$. Keeping (41) in mind, we obtain

Proposition 5.2. If $\rho \in(0,1)$ and $\sigma \in\left(0, \frac{1}{2}\right]$ satisfy $\rho+\sigma \leq 1$, then, for any $\tau \in(0, \rho)$ satisfying $\tau \leq \frac{1}{3}$ or $\tau+2 \sigma \leq 1$, an iteration step (37) and the refinement (38) can be implemented in $\mathcal{O}(N \log N)$ arithmetical operations.

Note that the case $\tau>\frac{1}{3}, \tau+2 \sigma \leq 1$ is possible only if $\sigma<\frac{1}{3}$, implying $\sigma<\tau$ and a band structure of $\mathbf{C}_{m}=\left(\mathbf{c}_{h j}\right)_{h, j \in \mathbb{Z}_{m}}$.

\section{Optimal COnVERGence of the methods}

Together with Propositions 5.1 and 5.2, the following theorem contains the main message of this paper.

Theorem 6.1. Let conditions (4) - (7) be fulfilled, and let $f \in H^{\mu+\alpha}, \mu+\alpha>\frac{1}{2}$. Let

$$
\begin{gathered}
n \sim N^{\rho}, \quad \frac{\mu+\alpha}{\mu+\alpha+\beta} \leq \rho<1, \\
l \sim N^{\sigma}, \quad 0<\sigma<1,
\end{gathered}
$$

and approximate the operators $\mathcal{A}$ and $\mathcal{B}\left(\right.$ see (2) and (16)) by $\mathcal{A}_{l}$ and $\mathcal{B}_{l}$ defined in (31) and (32). Then problem (1) has a unique solution $u=\mathcal{A}^{-1} f \in H^{\mu}$, and there exists an $N_{0}$ such that for $N \geq N_{0}$ the Galerkin method (35) provides a unique 
polynomial $u_{n} \in \mathcal{T}_{n}$, and for its refinement $u_{n, N} \in \mathcal{T}_{N}$ defined by (36) we have the error estimates

$$
\left\|u_{n, N}-u\right\|_{\lambda} \leq c_{\lambda, \mu} N^{\lambda-\mu}\|u\|_{\mu}, \quad-\alpha \leq \lambda \leq \mu .
$$

Furthermore, if we set

$$
m \sim N^{\tau}, \quad 0<\tau<\rho,
$$

there exists an $N_{1}$ such that for $N \geq N_{1}$ and any fixed $k$ satisfying

$$
k>\frac{1-\tau}{\tau} \frac{\mu+\alpha}{\beta}
$$

we have the error estimates

$$
\left\|u_{n, N}^{k}-u\right\|_{\lambda} \leq c_{\lambda, \mu} N^{\lambda-\mu}\|u\|_{\mu}, \quad-\alpha \leq \lambda \leq \mu,
$$

where $u_{n, N}^{k} \in \mathcal{T}_{n}$ is the refinement (38) of the two grid iteration approximation $u_{n}^{k}$ defined by (37).

Remark 6.1. For $u \in H^{\mu}$, error estimates (50) and (52) are of optimal order (compare with the error estimate of the orthogonal projection $P_{N} u$; see $(21)$ ).

Remark 6.2. Theorem 6.1 puts a condition only on $\rho$, leaving $\sigma$ and $\tau$ free. Choosing $\rho$ so that (49) is satisfied, the parameters $\sigma$ and $\tau$ should be submitted to the conditions of Propositions 5.1 and 5.2 to keep the implementation of the methods in $\mathcal{O}(N \log N)$ arithmetical operations.

Further remarks to Theorem 6.1 concerning conditions (49) and (51), and the saturation property of estimates (50) and (52) at $\lambda=-\alpha$, are presented after the proof of the theorem.

Proof of Theorem 6.1. Recall that $\mathcal{A} \in \mathcal{L}\left(H^{\lambda}, H^{\lambda+\alpha}\right), \mathcal{B} \in \mathcal{L}\left(H^{\lambda+\alpha}, H^{\lambda}\right)$ for any $\lambda \in \mathbb{R}$. We shall use the formulation (20) of problem (1):

$$
u=T u+g, \quad g=\mathcal{B} f, \quad T=I-\mathcal{B} \mathcal{A} .
$$

The operator $T \in \mathcal{L}\left(H^{\lambda}, H^{\lambda}\right)$ is actually bounded from $H^{\lambda}$ to $H^{\lambda+\beta}$ (see Lemma 3.1). Together with (21) this implies the inequalities

$$
\left\|\left(I-P_{n}\right) T\right\|_{\lambda, \lambda} \leq c_{\lambda} n^{-\beta}, \quad\left\|T\left(I-P_{n}\right)\right\|_{\lambda, \lambda} \leq c_{\lambda} n^{-\beta} .
$$

Let us represent the Galerkin equation (35) in a form similar to (53):

$$
u_{n}=P_{n} T_{l} u_{n}+P_{n} g_{l, n}, \quad g_{l, N}=\mathcal{B}_{l} Q_{N} f, \quad T_{l}=I-\mathcal{B}_{l} \mathcal{A}_{l} .
$$

As a consequence of (33) and (34),

$$
\left\|T_{l}-T\right\|_{\lambda, \lambda} \leq c_{\lambda, r} N^{-r} \quad \forall r>0, \lambda \in \mathbb{R} .
$$

It follows from (54) and (56) that $\left\|T-P_{n} T_{l}\right\|_{\lambda, \lambda} \rightarrow 0$ as $N \rightarrow \infty$. Since $T \in$ $\mathcal{L}\left(H^{\lambda}, H^{\lambda}\right)$ is compact and the homogeneous equation $v=T v$ has only the trivial solution (condition (7)), then $I-T$ is invertible in $H^{\lambda}$, and the same is true for $I-P_{n} T_{l}$ for sufficiently large $N$, say $N \geq N_{0}$. Thus the inverses are uniformly bounded:

$$
\left\|\left(I-P_{n} T_{l}\right)^{-1}\right\|_{\lambda, \lambda} \leq c_{\lambda}, \quad N \geq N_{0}, \quad \lambda \in \mathbb{R}
$$


For the solutions $u$ and $u_{n}$ of (53) and (55) we have

$$
\begin{aligned}
& \left(I-P_{n} T_{l}\right)\left(u_{n}-P_{n} u\right)=P_{n} g_{l, N}-P_{n} u+P_{n} T_{l} P_{n} u \\
& \quad=P_{n}\left(g_{l, N}-g\right)+P_{n}\left(T_{l}-T\right) P_{n} u-P_{n} T\left(I-P_{n}\right)\left(I-P_{n}\right) u,
\end{aligned}
$$

and due to (54), (56), and (57)

$$
\left\|u_{n}-P_{n} u\right\|_{\lambda} \leq c_{\lambda}\left(\left\|g_{l, N}-g\right\|_{\lambda}+N^{-r}\|u\|_{\lambda}+n^{-\beta}\left\|\left(I-P_{n}\right) u\right\|_{\lambda}\right), \quad \lambda \in \mathbb{R} .
$$

With the help of (22) and (34) we estimate

$$
\begin{aligned}
\left\|g_{l, N}-g\right\|_{\lambda} & \leq\left\|B_{l}\left(Q_{N} f-f\right)\right\|_{\lambda}+\left\|\left(B_{l}-B\right) f\right\|_{\lambda} \\
& \leq c_{\lambda}\left(\left\|Q_{N} f-f\right\|_{\lambda+\alpha}+N^{-r}\|f\|_{\lambda+\alpha}\right) \leq c_{\lambda, \mu} N^{\lambda-\mu}\|f\|_{\mu+\alpha} \\
& \leq c_{\lambda, \mu}^{\prime} N^{\lambda-\mu}\|u\|_{\mu}, \quad 0 \leq \lambda+\alpha \leq \mu+\alpha .
\end{aligned}
$$

Using also (21) and (54), we obtain

$$
\left\|u_{n}-P_{n} u\right\|_{\lambda} \leq c_{\lambda, \mu}\left(N^{\lambda-\mu}+n^{\lambda-\mu-\beta}\right)\|u\|_{\mu}, \quad-\alpha \leq \lambda \leq \mu,
$$

or, due to (49),

$$
\left\|u_{n}-P_{n} u\right\|_{\lambda} \leq c_{\lambda, \mu} N^{\lambda-\mu}\|u\|_{\mu}, \quad-\alpha \leq \lambda \leq \mu .
$$

Finally,

$$
\left\|\left(I-P_{n}\right) T u\right\|_{\lambda} \leq c_{\lambda, \mu} n^{\lambda-\mu-\beta}\|T u\|_{\mu+\beta} \leq c_{\lambda, \mu}^{\prime} N^{\lambda-\mu}\|u\|_{\mu}, \quad \lambda \leq \mu,
$$

For $u_{n, N}$ defined in (36) and the solution of (53) we have

$$
u_{n, N}-u=u_{n}-P_{n} u+\left(P_{N}-P_{n}\right)\left(g_{l, N}-g\right)-\left(P_{N}-P_{n}\right) T u-\left(I-P_{N}\right) u .
$$

From (58)-(60) and (21), the assertion (50) of the theorem follows.

Let us turn to the second part of the theorem. Represent (37) in the form

$$
u_{n}^{k}=S_{n} u_{n}^{k-1}+\phi_{n},
$$

where $S_{n}: \mathcal{T}_{n} \rightarrow \mathcal{T}_{n}$ and $\phi_{n} \in S_{n}$ are defined by

$$
\begin{aligned}
S_{n} & =I-\left(I-P_{m}\right) P_{n} \mathcal{B}_{l} \mathcal{A}_{l}-\left(P_{m} \mathcal{B}_{l} \mathcal{A}_{l}\right)^{-1} P_{m} \mathcal{B}_{l} \mathcal{A}_{l} \\
& =I-\left(P_{n}-P_{m}\right)\left(I-T_{l}\right)-\left(I-P_{m} T_{l}\right)^{-1} P_{m}\left(I-T_{l}\right) \\
& =\left(P_{n}-P_{m}\right) T_{l}+\left(I-P_{m} T_{l}\right)^{-1} P_{m} T_{l}\left(I-P_{m}\right), \\
\phi_{n} & =\left(P_{n}-P_{m}\right) g_{l, N}+\left(I-P_{m} T_{l}\right)^{-1} P_{m} g_{l, N} .
\end{aligned}
$$

It follows from (54), (56) and (57) that

$$
\left\|S_{n}\right\|_{\mu, \mu} \leq c_{\mu} m^{-\beta}, \quad N \geq N_{1}=N_{0} n / m .
$$

Using also (21), we obtain

$$
\left\|S_{n}\right\|_{\lambda, \mu} \leq c_{\alpha, \mu} m^{\lambda-\mu-\beta}, \quad-\alpha \leq \lambda \leq \mu, N \geq N_{1} .
$$

Here the constant $c_{\alpha, \mu}$ may be taken independent of $\lambda$, since the constants in (54), (56) and (57) may be taken uniformly bounded in $\lambda$ on every finite interval, the interval $[-\alpha, \mu]$ in our case. This can be seen from the interpolation theorem [11] for operators in scales of Hilbert spaces, $H^{\lambda}$ in our case. It is easy to check that for $u_{n}$, the solution of (35) and (55), we have $u_{n}=S_{n} u_{n}+\phi_{n}$; therefore

$$
u_{n}^{k}-u_{n}=S_{n}\left(u_{n}^{k-1}-u_{n}\right)=\ldots=S_{n}^{k}\left(u_{n}^{0}-u_{n}\right)=-S_{n}^{k} u_{n} .
$$


With the help of (61) and (62) we estimate

$$
\left\|u_{n}^{k}-u_{n}\right\|_{\lambda} \leq\left\|S_{n}\right\|_{\lambda, \mu}\left\|S_{n}\right\|_{\mu, \mu}^{k-1}\left\|u_{n}\right\|_{\mu} \leq c_{\alpha ; \mu}^{\prime} N^{\tau(\lambda-\mu-\beta)}\left(c_{\mu}^{\prime} N^{-\tau \beta}\right)^{k-1}\left\|u_{n}\right\|_{\mu} .
$$

For $k$ satisfying (51) this together with (59) yields

$$
\left\|u_{n}^{k}-u_{n}\right\|_{\lambda} \leq \epsilon_{k, N} N^{\lambda-\mu}\|u\|_{\mu}, \quad-\alpha \leq \lambda \leq \mu, \quad \epsilon_{k, N} \rightarrow 0 \quad \text { as } \quad N \rightarrow \infty .
$$

Since $u_{n, N}^{k}-u_{n, N}=u_{n}^{k}-u_{n}$, (52) follows from (50) and (63).

Remark 6.3. If $\rho \in(0,1)$ is not submitted to (49) then, nevertheless, (50) holds true for $\lambda_{0} \leq \lambda \leq \mu$ where $\lambda_{0}=\max \left(-\alpha, \mu-\beta \frac{\rho}{1-\rho}\right)$; to obtain (52) for these $\lambda$, (51) may be replaced by $k>\frac{1-\tau}{\tau} \frac{\mu-\lambda_{0}}{\beta}$

For instance, if $\alpha>0$ but we are interested in (50) and (52) only for $0 \leq \lambda \leq \mu$, then (49) and (51) may be replaced by the conditions $\frac{\mu}{\mu+\beta} \leq \rho<1$ and $k>\frac{1-\tau}{\tau} \frac{\mu}{\beta}$, respectively.

Let us illustrate the above results by means of the specific examples introduced in Section 1.

Example 6.1. We consider the biharmonic single layer equation introduced in Example 1.1, where $\alpha=3, \beta=1$. In this case the preconditioner $\mathcal{B}$ has the form

$(\mathcal{B} u)(t)=\frac{4 \pi^{2}}{\left|x^{\prime}(t)\right|^{3}}\left(\hat{u}(0)-\frac{4}{3} \hat{u}(1) e^{i 2 \pi t}-\frac{4}{3} \hat{u}(-1) e^{-i 2 \pi t}+\sum_{|l| \geq 2}|l|\left(l^{2}-1\right) \hat{u}(l) e^{i l 2 \pi t}\right)$.

Consider the solution $u=\mathcal{A}^{-1} f$ with the low-order smoothness property $u \in H^{0}$ (equivalently $f \in H^{3}$ ). Now, by Theorem 6.1 we have the approximate solution $u_{n, N} \in \mathcal{T}_{N}$ defined by (36) such that the optimal order estimates (50) are valid for $-3 \leq \lambda \leq 0$, if $\frac{3}{4} \leq \rho<1$ and $0<\sigma<1$. In order to compute the approximate solution in $O(N \log N)$ amount of work, we have to impose the additional condition $\sigma \leq \frac{1}{2}(1-\rho) \leq \frac{1}{8}$, if the direct solution by Proposition 5.1 is employed, and $\sigma \leq 1-\rho \leq \frac{1}{4}$ if the two grid iterations by Proposition 5.2 are involved, e.g., with $\tau=1 / 3$ (the optimal order of iterations will be achived for $k \geq 7$ ). Now consider a smoother exact solution, say, $u \in H^{6}$. In order to have an approximate solution (36) of optimal order of accuracy (50) for $0 \leq \lambda \leq 6$, we require $\rho \geq \frac{6}{7}$ by using Remark 6.3. To save the $O(N \log N)$ amount of work we need additionally $\sigma \leq \frac{1}{2}(1-\rho) \leq \frac{1}{14}$. This is realistic only for very large numbers $N\left(N^{1 / 14} \geq 2\right.$ if $N \geq 16384$ ). The requirements for the two-grid iteration are less stringent: with $\rho=\frac{6}{7}$ we choose $\tau=\frac{1}{3}, \sigma=\frac{1}{7}$. Then the iteration $u_{n, N}^{k}, k \geq 13$, is of optimal order for $0 \leq \lambda \leq 6$.

Example 6.2. Consider the singular integral equation of Example 1.2 assuming (6), (7). We have $\alpha=0$ and $\beta=\infty$, and the preconditioner takes the form $\mathcal{B}=$ $\frac{1}{a+b} P^{+}+\frac{1}{a-b} P^{-}$. In this case, where $\beta=\infty$, we have optimal order approximations $u_{n, N}, u_{n, N}^{k}$ defined by (36), (38) with any choices $0<\rho, \sigma<1,0<\tau<\rho$ (for $u_{n, N}^{k}$ with all $\left.k \geq 1\right)$. To have approximations with $O(N \log N)$ amount of work, the conditions of Propositions 5.1 or 5.2 have to be imposed on $\rho$ and $\sigma$.

Remark 6.4. The saturation of estimates (50) and (52) at $\lambda=-\alpha$ is caused by the saturation property (see (58) and (22)) $\left\|f-Q_{N} f\right\|_{\lambda+\alpha} \leq c_{\lambda, \mu} N^{\lambda-\mu}\|f\|_{\mu+\alpha}$ $(0 \leq \lambda+\alpha \leq \mu+\alpha)$ at $\lambda=-\alpha$. To obtain optimal order estimates (50) and (52) 
for $\lambda<-\alpha$, one has use other approximations of $g=\mathcal{B} f$ in $(35)-(38)$ rather than $g_{l, N}=\mathcal{B}_{l} Q_{N} f$.

We propose two possible approximations of $g$. Unlike $g_{l, N}=\mathcal{B}_{l} Q_{N} f$, these approximations are not so universal.

Remark 6.5. If the Fourier coefficients of $f$ are known we may put $g_{l, N}=\mathcal{B}_{l} P_{N} f$. In this case error estimates (50) and (52) hold for $\lambda_{0} \leq \lambda \leq \mu$ with arbitrary $\lambda_{0}, \mu \in \mathbb{R}, \lambda_{0}<\mu$, provided that

$$
f \in H^{\mu+\alpha}, \quad \frac{\mu-\lambda_{0}}{\mu-\lambda_{0}+\beta} \leq \rho<1, \quad k>\frac{1-\tau}{\tau} \frac{\mu-\lambda_{0}}{\beta} .
$$

No saturation of estimates (50) and (52) towards smaller $\lambda$ occurs.

Remark 6.6. Assume that, for some $j \in \mathbb{N}$, the integrations

$$
\begin{gathered}
c_{-k+1}=\int_{0}^{1} f^{(-k+1)}(s) d s, \quad f^{(-k)}(t)=\int_{0}^{t}\left[f^{(-k+1)}(s)-c_{-k+1}\right] d s, \\
k=1, \ldots, j,
\end{gathered}
$$

with $f^{(0)}(t)=f(t)$ can be carried out analytically (or at least the grid values of $f^{(-j)}$ are available with a sufficiently high accuracy). Put

$$
g_{l, N}=\mathcal{B}_{l}\left[\hat{f}(0)+(d / d t)^{j} Q_{N} f^{(-j)}\right] .
$$

In this case error estimates (50) and (52) hold for $\lambda_{0} \leq \lambda \leq \mu$ provided that (64) holds with $\mu+\alpha>\frac{1}{2},-\alpha-j \leq \lambda_{0}<\mu$.

Here the saturation of estimates (50) and (52) is reduced to $\lambda=-\alpha-j$.

Remark 6.7. Theorem 6.1 and Remarks 6.3-6.6 maintain their validity also for the Sloan type refinements

$$
u_{n, N}=T_{l} u_{n}+P_{N} g_{l, N}, \quad u_{n, N}^{k}=T_{l} u_{n}^{k}+P_{N} g_{l, N} \quad\left(T_{l}=I-\mathcal{B}_{l} \mathcal{A}_{l}\right)
$$

of $u_{n}$ and $u_{n}^{k}$ defined respectively by (35) and (37). Although these refinements are slightly more complicated than (36) and (38), they still cost $\mathcal{O}(N \log N)$ arithmetical operations submitting $\sigma$ and $\tau$ to conditions of Propositions 5.1 and 5.2.

\section{REFERENCES}

[1] B.A. Amosov, On the approximate solution of elliptic pseudodifferential equations on a smooth closed curve (in Russian), Z. Anal. Anwendungen 9(6) (1990), 545-563. MR 92g:35249

[2] B. Bialecki, Sinc-Nyström method for numerical solution of a dominant system of Cauchy singular integral equations given on a piecewise smooth contour, SIAM J. Numer. Anal. 26 (1989), 1194-1211. MR 90m:65225

[3] M. Costabel and J. Saranen, Boundary element analysis of a direct method for the biharmonic Dirichlet problem, Gohberg Anniversary Collection, vol. 2, Oper. Theory: Adv. Appl., Vol.41, Birkhäuser Verlag, Basel, 1989, 77-95. MR 90m:65195

[4] I. Gohberg and I. Feldman, Convolution equations and projection methods for their solution, AMS Translations of Mathematical Monographs 41, Amer. Math. Soc., Providence, 1974. MR 50:8149

[5] W. Hackbusch, Integralgleichungen, Teubner, Stuttgart, 1989. MR 90g:45001

[6] P. Henrici, Fast Fourier methods in computational complex analysis, SIAM Rev. 21 (4) (1979), 481-527. MR 80i:65031

[7] O. Kelle and G. Vainikko, A fully discrete Galerkin method for integral and pseudodifferential equations on closed curves, Z. Anal. Anwendungen 14 (1995), 593-622. MR 97c:65219 
[8] R. Kieser, B. Kleemann, and A. Rathsfeld, On a full discretization for a hypersingular boundary integral equation over smooth curves, Z. Anal. Anwendungen 11 (1992), 385-396. MR 94k:65186

[9] R. Kress, Linear integral equations, Springer Verlag, Berlin, Heidelberg, 1989. MR 90j: 45001

[10] R. Kress and I.H. Sloan, On the numerical solution of a logarithmic integral equation of the first kind for the Helmholtz equation, Numer. Math. 66 (1993), 199-214. MR 95d:65108

[11] J.L. Lions and E. Magenes, Non-homogeneous boundary value problems and applications I, Springer Verlag, Berlin, Heidelberg, New York, 1972. MR 50:2670

[12] W. McLean, S. Prössdorf, and W.L. Wendland, Pointwise error estimates for the trigonometric collocation method applied to singular integral equations and periodic pseudodifferential equations, J. Integral Equations Appl., 2(1) (1989), 125-146. MR 91f:65218

[13] W. McLean, S. Prössdorf, and W.L. Wendland, A fully discrete trigonometric collocation method, J. Integral Equations Appl. 5(1) (1993), 103-129. MR 94m:65203

[14] W. McLean and W.L. Wendland, Trigonometric approximation of periodic pseudodifferential equations, The Gohberg Anniversary Collection, Vol. 2, Oper. Theory: Adv. Appl., Vol.41, Birkäuser Verlag, Basel, 1989, 359-383. MR 91f:65217

[15] S.G. Mikhlin and S. Prössdorf, Singular integral operators, Springer Verlag, Berlin, 1986. MR 88e: 47097

[16] S. Prössdorf and J. Saranen, A fully discrete approximation method for the exterior Neumann problem of the Helmholtz equation, Z. Anal. Andwendungen 13 (1994), 683-695. MR 95i:65181

[17] L. Reichel and Y. Yan, Fast solution of a class of periodic pseudodifferential equations, J. Integral Equations Appl. 6 (1994), 401-426. MR 96b:65118

[18] J. Saranen and L. Schroderus, Quadrature methods for strongly elliptic equations of negative order on smooth closed curves, SIAM J. Numer. Anal. 30(6) (1993), 1769-1795. MR 94k:65187

[19] J. Saranen and G. Vainikko, Two-grid solution of Symm's integral equation, Math. Nachr. 177 (1996), 265-279. MR 97f:65076

[20] J. Saranen and G. Vainikko, Trigonometric collocation methods with product integration for boundary integral equations on closed curves, SIAM J. Numer. Anal. 33(4) (1996), 15771596. MR 97m:65245

[21] F. Stenger, Numerical methods based on sinc and analytic functions, Springer Verlag, New York, Berlin, 1993. MR 94k:65003

University of Oulu, Department of Mathematical Sciences, 90570 Oulu Finland

E-mail address: jsaranen@cc.oulu.fi

Institut of Mathematics, Helsinki University of Technology, 02150 Espoo, Finland

E-mail address: gennadi.vainikko@hut.fi 